\title{
The Siting and Sizing Problem of Distributed Generation Based on a Novel Fractional Particle Swarm Optimization Algorithm
}

\author{
Fengming Zhang ${ }^{1}$, Lingyan Que $^{1}$, Xinxin Zhang ${ }^{1}$, Fumian Wang,,*, and Bing Wang ${ }^{2}$ \\ ${ }^{1}$ Shaoxing Power Supply Company, State Grid Zhejiang Electric Power Corporation, 312000 Shaoxing, China \\ ${ }^{2}$ College of Energy and Electrical Engineering, Hohai University, 211100 Nanjing, China
}

\begin{abstract}
Large-scale distributed generation grid-connection brings huge economic and environmental benefits, but also threatens the stability of the grid. To make the grid consume a higher proportion of distributed generation, it is necessary to optimize the location and capacity of the distributed generation connected to the grid. Firstly, the uncertainty analysis model of wind speed, illumination intensity, and load of grid is established. Secondly, a distributed generation location and capacity planning model with the lowest annual comprehensive cost as the objective function is constructed. Then, a novel fractional particle swarm optimization algorithm is proposed, and the performance of the algorithm on complex optimization problems is tested. Finally, the simulation results of the IEEE 33-bus system example verify the rationality of the established model and the effectiveness of the proposed algorithm.
\end{abstract}

\section{Introduction}

Due to the limited reserves of fossil fuels and the increasingly severe environmental problems brought about by them, the realization of low-carbon and sustainable development has become the core concept of solving energy problems. Compared with traditional thermal power generation, distributed generation (DG) including wind turbine generator (WTG) and photovoltaic generator (PVG) has clean and sustainable characteristics. DG connected with the grid can bring huge economic and environmental benefits [1]. However, the inherent stochastic and fluctuating characteristics of DG's output will have an impact on the grid. Large-scale DG gridconnection will result in a decrease in power quality and power supply reliability [2]. The location and capacity of DG connected to the distribution grid will affect the grid's network topology and power flow of the grid [3]. Therefore, optimizing the location and capacity of DG can improve the level of new energy consumption in the distribution grid, the stability of the distribution system, and economic benefits.

At present, the research on the siting and sizing problem of DG is mainly focused on dealing with the uncertainties of wind speed, illumination intensity and load of grid. Reference [4] proposed a probabilistic power flow calculation method considering the correlation of input variables. This algorithm has the advantages of high accuracy and fast speed. Reference [5] established an optimization model with the minimum annual comprehensive cost as the objective function. The Latin hypercube sampling method was used to generate the initial scene, and the improved synchronous backgeneration reduction method was used to reduce the scene.
Reference [6] established an intermittent distributed generation site selection and capacity planning model based on the opportunity-constrained programming method, and used a rank correlation coefficient matrix to characterize the correlation between random variables. Reference [7] included environmental factors into the objective function, making the planning results closer to the actual project. The above-mentioned documents fully considered the uncertainty and correlation of the input variables of the system, and constructed the corresponding DG site selection and capacity planning model, but did not solve the problem of the parameter selection of the model solution algorithm, and it is easy to locally converge.

The mathematical model of DG's location and capacity programming is a kind of high-dimensional non-convex optimization problem with multiple constraints. Traditional convex optimization methods are difficult to deal with complex and variable constraints. Therefore, intelligent optimization algorithms are widely used to solve the above problems. The traditional Particle Swarm Optimization (PSO) algorithm is a representative swarm optimization algorithm, but it has the shortcomings of easy local convergence and low convergence accuracy. Since Lévy disturbance has the characteristic of large jump sizes, the additive Lévy disturbance can make the particle have a wider search range. On this basis, different from the multiplicative random disturbance in the traditional PSO, the Lévy disturbance is introduced in the form of additive random disturbance. The Fractional Particle Swarm Optimization (FPSO) algorithm is proposed in this paper.

This paper comprehensively considers the uncertainty and correlation of wind speed, illumination intensity, and load, and establishes a DG site selection and capacity planning model based on opportunity constraints with the

\footnotetext{
*Corresponding author: fumian98@foxmail.com
} 
lowest comprehensive annual cost as the objective function, and proposes FPSO to solve the model. The planning model was simulated on the IEEE 33-bus system.

\section{Planning Model}

\subsection{Uncertainty modelling}

\subsubsection{Uncertainty of WTG's output power}

It is generally considered that wind speed is the main factor affecting the output power of WTG. The law of wind speed generally follows the two-parameter Weibull distribution [8], and its probability density function is:

$$
f(V)=\left(\frac{k}{c}\right)\left(\frac{V}{c}\right)^{k-1} \exp \left[-\left(\frac{V}{c}\right)^{k}\right]
$$

where $V$ is the actual wind speed while $k$ and $c$ are the shape parameter and scale parameter, respectively.

The wind speed can be converted into the output power of WTG through the WTG power model, which can be formulated as:

$$
P_{W T G}= \begin{cases}0 & V \leq V_{c i} \text { or } V \geq V_{c o} \\ \frac{V^{3}-V_{c i}^{3}}{V_{r}^{3}-V_{c i}^{3}} P_{W T G, r} & V_{c i} \leq V<V_{r} \\ P_{W T G, r} & V_{r} \leq V<V_{c o}\end{cases}
$$

where $V_{c i}, V_{r}$, and $V_{c o}$ denote the cut-in wind speed, the rated wind speed, and the cut-out wind speed, respectively. $P_{W T G, r}$ is the rated output power of WTG.

\subsubsection{Uncertainty of PVG's output power}

A lot of studies have shown that the randomness of illumination intensity is usually described by Beta distribution [9], and its probability density function is:

$$
f(S)=\frac{\Gamma(\alpha+\beta)}{\Gamma(\alpha) \Gamma(\beta)}\left(\frac{S}{S_{r}}\right)^{\alpha-1}\left(1-\frac{S}{S_{r}}\right)^{\beta-1}
$$

where $S$ is the illumination intensity, $S_{r}$ is the rated value, $\alpha$ and $\beta$ are two shape parameters of Beta distribution, and $\Gamma(\cdot)$ denotes the Gamma function.

The relationship between actual PVG's output and illumination intensity can be approximated as follows [9]:

$$
P_{P V}= \begin{cases}P_{P V, r} \frac{S}{S_{r}} & I \leq S_{r} \\ P_{P V, r} & I>S_{r}\end{cases}
$$

where $P_{P V, r}$ is the rated power of PVG and $S_{r}$ is the rated illumination intensity.

\subsubsection{Uncertainty of load}

Normal distribution is generally used to describe the uncertainty of load size [9] :

$$
\begin{aligned}
& f\left(P_{L, i}\right)=\frac{1}{\sqrt{2 \pi} \sigma_{P, i}} \exp \left(-\frac{\left(P_{L, i}-\mu_{P, i}\right)^{2}}{2 \sigma_{P, i}^{2}}\right) \\
& f\left(Q_{L, i}\right)=\frac{1}{\sqrt{2 \pi} \sigma_{Q, i}} \exp \left(-\frac{\left(P_{Q, i}-\mu_{Q, i}\right)^{2}}{2 \sigma_{Q, i}^{2}}\right)
\end{aligned}
$$

where $P_{L, i}$ and $Q_{L, i}$ are the active and reactive power load at bus $i$, and $\mu_{P, i}, \mu_{Q, i}$ and $\sigma_{P, i}, \sigma_{Q, i}$ are, respectively, the mean value and standard deviation of the active and reactive power load at bus $i$.

\subsubsection{Uncertainty treatment method}

Samples conforming to the characteristics of random variables of the power system are selected from the probability distribution of the uncertain model, and the distribution characteristics of output variables can be used as a typical scenario of system operation [10]. QuasiMonte Carlo Simulation (QMCS) is a sampling method that replaces the random number sequence in Monte Carlo method by generating low differentiation sequence in high dimensional space, which can avoid the spatial inhomogeneity of Monte Carlo sampling. At the same time, because QMCS generates all the sampling points at one time, compared with Monte Carlo, which generates a large number of random numbers in each iteration, it also has a great improvement in the time efficiency of the algorithm.

After the sampling points are obtained through QMCS, it is necessary to transform the sampling points into a sample sequence conforming to the probability distribution of the random variables of the system. Johnson transform can establish the relationship between normal distribution and its own distribution based on the target variables' mean value, variance, skewness, kurtosis and correlation [11]. First, the sampling points are converted to independent normal distribution by the inverse operation of the cumulative function equation of normal distribution, and then the original sampling data of the system random variables are obtained by Johnson transform.

\subsection{Deterministic optimization model}

\subsubsection{Objective function}

The model takes the minimum annual comprehensive cost of DG site selection and capacity as the optimization goal. The annual comprehensive costs mainly include DG investment costs $C_{I}$, DG operation and maintenance costs $C_{O M}$, distribution network active power loss costs $C_{L}$, and full consideration of distribution network power purchase costs $C_{P}$ and government subsidies $C_{S}$. Equivalently amortize the DG investment cost to each year of the DG life cycle through the current value conversion coefficient of equivalent years:

$$
\tau=d(1+d)^{y} /\left((1+d)^{y}-1\right)
$$


where $d$ is the discount rate, and $y$ is the number of DG life period years.

The objective function is as follows:

$$
\begin{aligned}
\min C= & C_{I}+C_{O M}+C_{L}+C_{P}-C_{S} \\
= & \tau \sum_{i \in \Omega_{n}} \sum_{j \in \Omega_{r}}\left(c_{D G, i}^{j} c_{P}^{j} P_{D G}^{j}\right)+\sum_{i \in \Omega_{n}} \sum_{j \in \Omega_{r}}\left(c_{O M}^{j} E_{D G, i}^{j}\right) \\
& +\sum_{l \in \Omega_{s}}\left(c_{e} P_{L o s s,} T_{\max }\right)+\sum_{m \in \Omega_{g}}\left(c_{e} E_{G E N, m}\right) \\
& -\sum_{i \in \Omega_{n}} \sum_{j \in \Omega_{r}}\left(c_{f} E_{D G, i}^{j}\right)
\end{aligned}
$$

where $\Omega_{n}, \Omega_{r}, \Omega_{b}$, and $\Omega_{g}$ are the bus set of DG, type set of DG, branch set of grid and generator bus set of grid, respectively. $c_{D G, i}^{j}$ is the number of DG of type $j$ installed at bus $i$, while $c_{P}^{j}$ is the unit price of DG of type $j$ and $P_{D G}^{j}$ represents the unit capacity of DG of type $j$. $c_{O M}^{j}$ is the unit price of DG's generated energy, while $c_{e}$ is the price of electrovalence. $P_{\text {Loss }, l}$ is the active power loss at branch $l$ under the maximum load. $T_{\max }$ is the maximum load hours per year. $E_{G E N, m}$ is the generated energy at bus $m \cdot c_{f}$ is the government subsidies cost per DG unit of electricity generation, while $E_{D G, i}^{j}$ is the generation of type $j$ DG at bus $i$.

\subsubsection{Constraints}

The constraints of the model include power flow constraint, bus voltage constraint, branch current constraint and DG's capacity constraint.

1)Power flow constraint

$$
\left\{\begin{array}{l}
P_{i}-U_{i} \sum_{j \in \Omega_{d}} U_{j}\left(G_{i j} \cos \theta_{i j}+B_{i j} \sin \theta_{i j}\right)=0 \\
Q_{i}-U_{i} \sum_{j \in \Omega_{d}} U_{j}\left(G_{i j} \sin \theta_{i j}-B_{i j} \cos \theta_{i j}\right)=0
\end{array} \quad \forall i, j \in \Omega_{d}\right.
$$

where $\Omega_{d}$ is the bus set of grid. $P_{i}, Q_{i}$, and $U_{i}$ are respectively the active power, reactive power and voltage at bus $i$, while $G_{i j}, B_{i j}$, and $\theta_{i j}$ are respectively the conductance, susceptance, and phase angle difference between bus $i$ and bus $j$.

2)Oppotunity constraint of bus voltage

$$
P\left\{\underline{U}_{i} \leq U_{i} \leq \bar{U}_{i}\right\} \leq \alpha_{U} \quad \forall i \in \Omega_{d}
$$

where $P(A)$ represents the probability that event $A$ is true, while $\alpha_{U}$ is the confidence level satisfying the bus voltage constraint. $\underline{U}_{i}$ and $\bar{U}_{i}$ are the upper and lower limit of voltage at bus $i$.

3)Branch current constraint

$$
P\left\{I_{j} \leq \bar{I}_{j}\right\} \leq \alpha_{I} \quad \forall j \in \Omega_{b}
$$

where $\alpha_{I}$ is the confidence level satisfying the branch current constraint. $\bar{I}_{j}$ is the upper limit of current through branch $j$.

4)DG's capacity constraint

$$
\left\{\begin{aligned}
\sum_{i \in \Omega_{n}} \sum_{j \in \Omega_{r}}\left(c_{D G, i}^{j} P_{D G}^{j}\right) \leq \bar{P}_{D G} \\
c_{D G, i}^{j} P_{D G}^{j} \leq \bar{P}_{D G, i}^{j} \quad \forall i \in \Omega_{n} j \in \Omega_{r}
\end{aligned}\right.
$$

where $\bar{P}_{D G}$ is the upper limit of DG's capacity in grid, while $\bar{P}_{D G, i}^{j}$ is the upper limit of type $j$ DG's capacity at bus $i$.

\section{FPSO algorithm}

\subsection{PSO algorithm}

Particle swarm optimization algorithm is an intelligent optimization algorithm based on swarm search [12], which is widely used in large-scale complex optimization problems. Its process can be formulated as :

$$
\left\{\begin{array}{l}
v_{i}^{k+1}=v_{i}^{k}-\rho_{1}\left(x_{i}^{k}-x_{b}\right)-\rho_{2}\left(x_{i}^{k}-x_{i}^{p}\right) \\
x_{i}^{k+1}=x_{i}^{k}+v_{i}^{k+1}
\end{array}\right.
$$

where $i=1,2, \ldots, N$ represents the $i$ th particle in the population, while $v_{i}^{k}$ and $x_{i}^{k}$ are respectively the velocity and position of the particle in the $k$ th iteration. $\rho_{1}, \rho_{2}>0$ are two independent random disturbance. $x_{b}$ is the best position in the solution space that the particle groups have been to, while $x_{i}^{p}$ is the best of particle $i . \rho_{1}\left(x_{i}^{k}-x_{b}\right)$ represents the global search ability of particle $i$, while $\rho_{2}\left(x_{i}^{k}-x_{i}^{p}\right)$ represents the local search ability. The working mechanism of both is similar to providing gradient descent information toward a given target.

Many swarm-based search algorithms are inspired by PSO, such as Cuckoo algorithm, Bat algorithm and Differential Evolution algorithm. The global search strategy of these algorithms can be formulated as:

$$
x_{i}^{k+1}=x_{i}^{k}-\rho_{k}\left(x_{i}^{k}-x_{b}\right)
$$

where $\rho_{k}$ is the randomness in the $k$ th iteration. We have to mention that (13) is an accelerated form of (14).

\subsection{Lévy disturbance}

In the PSO algorithm, the searching ability of particles comes from stochastic perturbation and historical information, and the perturbation can accelerate the convergence speed of the algorithm. The form of randomness is arbitrary. The common form of perturbation distribution in the algorithm includes uniform distribution and normal distribution. When the objective function has saddle points and multiple minimum points, the traditional PSO algorithm is easy to fall into the local optimum because of the limitation of the perturbation form.

Consider a symmetric form of Lévy stable distribution $L(x)$, and its characteristic function is:

$$
E\left\{e^{i k x}\right\}=\exp \left\{-\sigma^{\alpha}|k|^{\alpha}\right\}
$$


where $\sigma>0$ is the scale parameter of $L(x)$ and $0<\alpha \leq 2$ is the order. When $\alpha=2, L(x)$ reduce to a normal distribution.

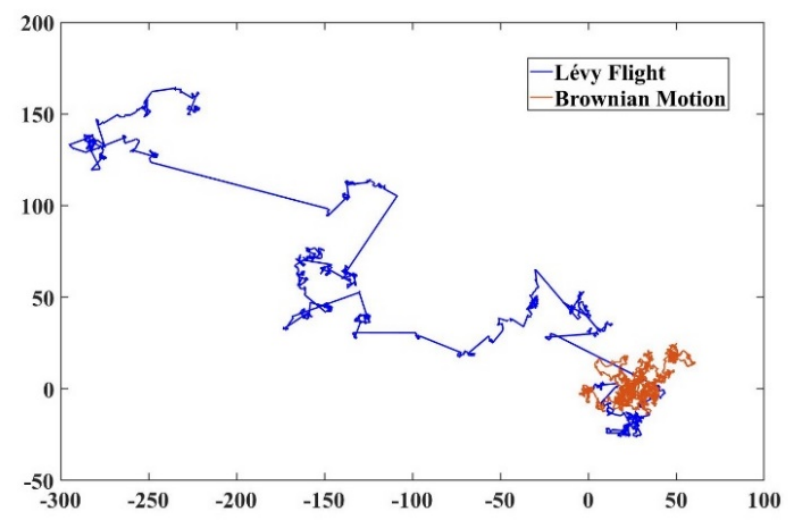

Figure 1. Comparison of Lévy flight and Brownian motion

Lévy flight is a form of large size jump caused by Lévy disturbance. Compared with the Brownian motion where the disturbance increment follows the normal distribution, Lévy flight has a larger search space. This process is illustrated visually as follows: in a two-dimensional plane, two particles with initial positions are generated. The step size of one particle is generated from the standard normal distribution, which represents Brownian motion. And the step size of the other particle is generated from the symmetric standard Lévy distribution with order and scale, representing Lévy flight. The direction of motion of the two particles is generated from the uniform distribution between. that

The particles' trajectory is shown in Figure 1. We have

1) The range of Brownian motion is near the initial position, which means that the particle only reaches the local region in the search process.

2) During Lévy flight, the particle jumps frequently, which makes the particle have a wider search range than Brownian motion.

\subsection{Some discussions on FPSO}

\subsubsection{Multiplicative randomness or additive randomness}

For the algorithm defined in (14), randomness exists in the form of multipliers in the population iteration. Consider two extreme cases:

a) Assuming that all particles have the same initial value, the population will not be updated at this time and fall into the initial position.

b)Assuming that the population has only two particles, the search direction of the particles will only be on the line between the two.

If during the population iteration, the randomness exists in the form of an addend:

$$
x_{i}^{k+1}=x_{i}^{k}-\rho_{k}\left(x_{i}^{k}-x_{b}\right)+\varepsilon \eta_{i}^{k}
$$

where $\varepsilon$ is the scale parameter, while $\eta_{i}^{k}$ is randomness of a given distribution. With such additive randomness:
a)Even if all particles have the same initial value, the population will be updated.

b)The particles would still search a wide range to find a feasible solution if the population has only two particles.

\subsubsection{The impact of the number of particles on the performance of algorithm}

In the traditional particle swarm algorithm, due to the poor global optimization ability of particles, a certain number of populations are needed to complete the optimization process. However, the fractional particle swarm algorithm greatly reduces the number of particles required due to the large step size jump of the particles, so that the number of iterations of the algorithm is greatly reduced, and the performance of the algorithm is effectively improved.

\subsubsection{Initial position insensitivity}

The traditional particle swarm algorithm has a small optimization range, so it is necessary to distribute the particles as evenly as possible in the solution space at the beginning of the population iteration. But for some largescale nonlinear optimization problems, it is difficult to determine the range of the solution space or the dimensionality hazard problem caused by the huge number of optimization variables. The large step size jump of the fractional particle swarm algorithm in the iterative process can avoid the difficulty of selecting the initial value of the population. Even if all the particles are placed in a fixed initial position, the particles can still achieve a large-scale optimization.

\section{Case studies}

The simulation of solving DG planning model using FPSO is carried out on the IEEE 33-bus system, and IEEE 33bus system data reference [15]. The relevant planning data is now explained: the planning period is 20 years, the discount rate is 0.1 , and the candidate nodes for DG installation in the distribution network are nodes $3,6,7$, $13,17,19$ and 31 , each of which is to be selected The upper limit of the node's DG installation capacity is $800 \mathrm{~kW}$. There are two types of DG: WTG and PVG. Related parameters of WTG: rated capacity of $50 \mathrm{~kW}$, $V_{c i}=3.5 \mathrm{~m} / \mathrm{s}, V_{r}=12 \mathrm{~m} / \mathrm{s}, V_{c o}=20 \mathrm{~m} / \mathrm{s}$; related parameters of PVG: rated capacity of $50 \mathrm{~kW}, S_{r}=1000 \mathrm{~W} / \mathrm{m} 2$. The wind speed in this area obeys the Weibull distribution with parameters $k=1.83$ and $c=9.93$, and the light intensity obeys the Beta distribution with parameters $\alpha=2.06$, $\beta=2.5$. WTG investment and operation and maintenance costs are 10000 yuan $/ \mathrm{kW}$ and 0.33 yuan $/ \mathrm{kWh}$, respectively, and PVG investment and operation and maintenance costs are 13000 yuan $/ \mathrm{kW}$ and 0.1 yuan $/ \mathrm{kWh}$, respectively. The load follows a Gaussian distribution, and the standard deviation is $10 \%$ of the mean. The fluctuation range of the node voltage is 0.9-1.1 (standard unit value), and the upper limit of the current of each line can be found in literature [15], confidence level $\alpha_{U}=\alpha_{I}=0.95$. The electricity price is 0.7 yuan $/ \mathrm{kWh}$, the government subsidy is 0.25 
yuan/kWh, and the annual maximum load hours is 4200 hours.

Aiming at the economic optimization problem of DG location and capacity described in section 2.2 , the traditional PSO and the FPSO mentioned in this paper are used to solve the problem. The results are shown in Table 1, Table 2 and Figure 2. Among them, Table 1 is the solution result of the DG installation location and capacity, and compares the two with the annual comprehensive cost of the distribution network without DG; Table 2 is the composition of the annual comprehensive cost; Figure 2 is a visual description of the solution result.

Table 1. The result of the case

\begin{tabular}{|c|c|c|}
\hline Algorithm & Location and Capacity & $\begin{array}{c}\text { Costs } / \\
(10,000 \mathrm{CNY})\end{array}$ \\
\hline No DG & None & 931.6664 \\
\hline PSO & $\begin{array}{c}3(1,1), 6(3,1), 7(3,2), \\
13(3,1), 17(2,1), 19(3, \\
1), 31(2,2)\end{array}$ & 898.0473 \\
\hline FPSO & $\begin{array}{c}13(7,0), 17(1,3), \\
31(10,5)\end{array}$ & 892.2336 \\
\hline
\end{tabular}

NOTE: 3(1, 1) means that there are 1 WTG and 1 PVG at bus 3.

Table 2. Composition of the annual comprehensive cost

\begin{tabular}{|c|c|c|c|}
\hline $\begin{array}{c}\text { Parts } \\
/(10,000 \mathrm{CNY})\end{array}$ & No DG & PSO & FPSO \\
\hline$C_{I}$ & 0 & 168.5546 & 166.7927 \\
\hline$C_{O M}$ & 0 & 73.5596 & 75.6952 \\
\hline$C_{L}$ & 41.6899 & 36.9013 & 35.7371 \\
\hline$C_{P}$ & 889.9765 & 686.4820 & 681.7741 \\
\hline$C_{S}$ & 0 & 67.4502 & 67.7655 \\
\hline Total & 931.6664 & 898.0473 & 892.2336 \\
\hline
\end{tabular}

It can be seen from Table 1 that the optimization result of FPSO is better than that of PSO. Judging from the cost composition in Table 2, the access of DG has reduced the cost of active power loss of the distribution network, and the cost of purchasing power from the upper-level grid has been greatly reduced by the distribution network. It can be concluded that the access of DG is conducive to system energy saving and emission reduction. From the point of view of the location of the DG in the optimization results, since the IEEE 33-bus system is a radial distribution grid, installing DG at a bus far away from the upper-level grid is beneficial to the stability of the grid voltage.

The comparison of the algorithm convergence characteristics is shown in Figure 3. It can be seen that the PSO has fallen into the local optimum during the optimization process, while the FPSO has jumped out of the local optimum many times due to the large step jumps of Lévy flight, thereby obtaining better optimization results.

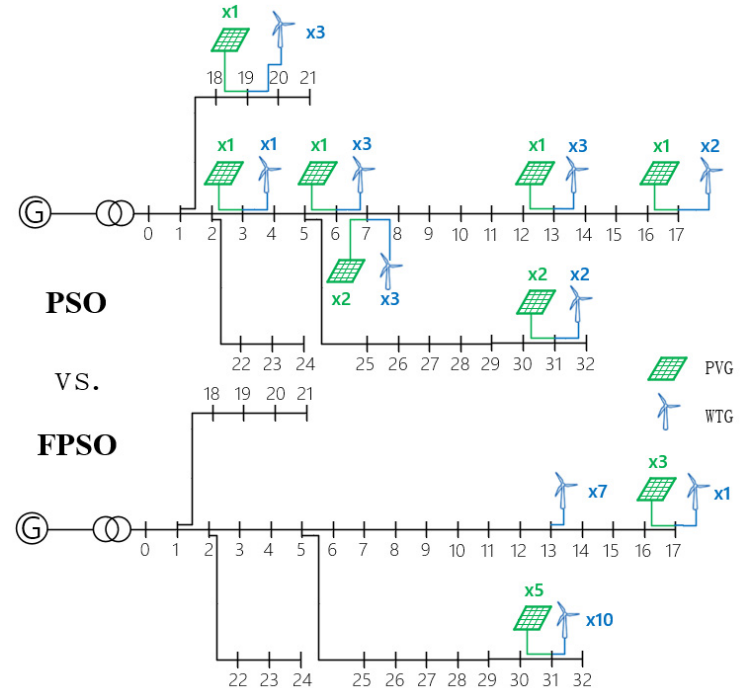

Figure 2. The location and capacity of DG in IEEE 33-bus system

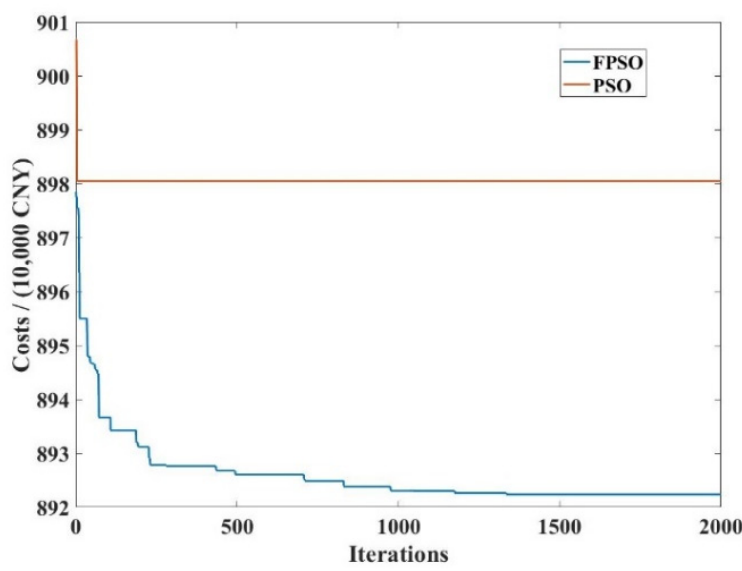

Figure 3. Convergence characteristics comparison of PSO and FPSO

\section{Conclusion}

Under the premise of DG and load uncertainty modeling, this paper aims to optimize the economy of the distribution network, and establishes a DG location and capacity planning model based on opportunity constraints. Aiming at the limitations of traditional PSO for solving large-scale optimization problems, the FPSO is proposed to solve the above model. FPSO has obtained a more economical DG configuration result in the IEEE 33-bus system, from which the following conclusions can be drawn:

a) The access of DG reduces the active network loss of the system, reduces the comprehensive annual cost of the system, and improves the economy of the system.

b)Optimizing the location and capacity of DG can improve the level of renewable energy consumption in the distribution network, which is conducive to the realization of low-carbon and green production of electric energy.

c)Compared with PSO, FPSO has higher solving efficiency and convergence accuracy on complex optimization problems, and it can avoid the difficulty of selecting the initial value of PSO. 


\section{Acknowledgments}

The work described in this paper was fully supported by the Project of State Grid Zhejiang Electric Power Corporation (NO. 5211SX1900PR): Research and application of key mining technologies based on the big data of full time and space regulation.

\section{Reference}

1. S.X. Fan, T.J. Pu, T.Y. Liu, et al, Transactions of China Electrotechnical Society, 31(2), 92-101 (2016)

2. H. Xiao, W. Pei, W. Deng, et al, Transactions of China Electrotechnical Society, 31(1), 203-213 (2017)

3. J. Mitra, M.R. Vallem, C. Singh, IEEE Transactions on Industry Applications, 52(3), 1989-1997 (2016)

4. Y. Chen, J.Y. Chen, S.J. Cheng, Proceedings of the CSEE, 31(22), 80-87 (2011)

5. Z.Q. Cao, M.F. Peng, M.E. Shen, Proceedings of the CSU-EPSA, doi: 10.19635/j.cnki.csu-epsa.000508 (2021)

6. S.X. Zhang, K.Li, H.Z. Cheng, et al, Automation of Electric Power Systems, 39(8), 53-58 (2015)

7. Z. Chu, Z. Li, W.W. Bai, Power System Protection and Control, 45(13), 34-41 (2017)

8. X.S. Zhang, Y. Yuan, Z. Chen, et al, Power System Technology, 36(06), 195-199 (2012)

9. S. Zhang, H. Cheng, L. Zhang, et al, IEEE Transactions on Power Systems, 28(3), 3215-3225 (2013)

10. X.Y. Ma, Scenario Analysis and Stochastic Programming of Wind-Integrated Power Systems (Wuhan University, 2014)

11. T. Soukissian, Applied Energy, 111, 982-1000 (2013)

12. J. Kennedy, R. Eberhart, International Conference on Neural Networks (Perth, Australia, 1995)

13. A.C. Wilson, B. Recht, M.I. Jordan, arXiv: 1611.02635 (2016)

14. D. Karaboga, B. Akay. Applied Mathematics and Computation, 214(1), 108-132 (2009)

15. M.E. Baran, F.F. Wu, IEEE Power Engineering Review, 9(4), 101-102 (1989) 